\title{
SUPERPURE DIGRAPH DESIGNS
}

\author{
SVEN HARTMANN
}

\begin{abstract}
A digraph design is a decomposition of a complete (symmetric) digraph into copies of pre-specified digraphs. Well-known examples for digraph designs are Mendelsohn designs, directed designs or orthogonal directed covers. A digraph design is superpure if any two of the subdigraphs in the decomposition have no more than two vertices in common. We give an asymptotic existence theorem for superpure digraph designs, which generalizes an earlier result of Lamken and Wilson. As an immediate consequence, we obtain new results for supersimple designs and pure perfect Mendelsohn designs.
\end{abstract}

Keywords: decomposition, digraph design, pure design, Mendelsohn design 1991 Mathematical Subject Classification: 05C70, 05B40

\section{INTRODUCTION}

We begin with some definitions from graph and design theory. A family $\mathcal{F}$ of subdigraphs (called blocks) of a given digraph $H$ forms a $(\mathcal{G}, \lambda)$ decomposition of $H$ if every arc of $H$ occurs in exactly $\lambda$ blocks and every block is isomorphic to some member of a pre-specified family $\mathcal{G}$ of digraphs. For every positive integer $v$, let $D_{v}$ denote the complete (symmetric) digraph with $v$ vertices. Decompositions of complete digraphs are also known as digraph designs. A $(v, \mathcal{G}, \lambda)$ digraph design is a $(\mathcal{G}, \lambda)$ decomposition of $D_{v}$. If $\mathcal{G}$ consists of a single digraph $G$ only, we write $(v, G, \lambda)$, for short.

Digraph designs are common generalizations of graph designs, Mendelsohn designs and directed designs, which have been widely studied in literature since the early seventies. A $(v, k, \lambda)$ block design is a family $\mathcal{F}$ of $k$-element subsets (called blocks) of a $v$-element set $V$ such that every pair of distinct elements of $V$ is contained in precisely $\lambda$ blocks. In 1971, Mendelsohn [31] extended the standard notion of a block design by allowing blocks to contain ordered rather than unordered pairs. These designs are now more commonly called Mendelsohn designs due to Mathon and Rosa [29]. A Mendelsohn design is just a $\left(v, C_{k}, \lambda\right)$ digraph design, where $C_{k}$ is the directed $k$-cycle, see Figure 1.

Later, a similar idea was used by Huang and Mendelsohn [24] who studied directed designs. A directed design is a $\left(v, T_{k}, \lambda\right)$ digraph design where $T_{k}$ is the transitively

Date: June 10, 2000.

Address: FB Mathematik, Universität Rostock, 18051 Rostock, Germany.

E-mail address: sven.hartmann@mathematik.uni-rostock.de. 
directed $k$-tournament, see Figure 1. For an overview on Mendelsohn and directed designs, we refer to $[3,12,30]$.

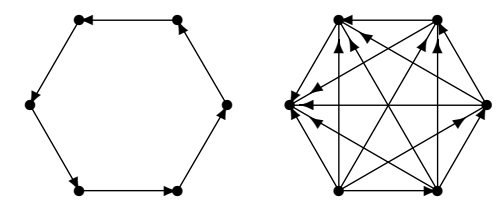

Figure 1. The digraphs $C_{k}$ and $T_{k}$, for $k=6$.

On the other hand, Hell and Rosa [22] as well as Bermond and Sotteau [6] generalized the notion of a block design by allowing the blocks to take the structure of a graph. These designs are known as graph designs (or $G$-designs). Let $S$ be a symmetric digraph. Then a $(v, S, \lambda)$ digraph design becomes a graph design when replacing every block by its underlying (undirected) graph. A $(v, k, \lambda)$ block design, in particular, is equivalent to a $\left(v, D_{k}, \lambda\right)$ digraph design. For an overview on graph designs, we refer to $[8,21,34]$.

Let $G$ be a digraph with vertex set $V(G)$ and arc set $E(G)$. By $\epsilon(G)$ we denote the number of arcs in $G$, and by $\delta_{u}^{+}(G), \delta_{u}^{-}(G)$ the in- and outdegree of a vertex $u$ in $G$. It should be noted, that all digraphs considered in this paper are finite and without loops or multiple arcs.

In Bosák's book [8], we also find necessary conditions for the existence of digraph designs. A $(v, G, \lambda)$ digraph design obviously has $\lambda \epsilon\left(D_{v}\right) / \epsilon(G)$ blocks. Hence, $\lambda v(v-1)$ is divisible by $\epsilon(G)$. A second necessary condition may be obtained by studying the vertex degrees in $G$. It is straightforward to see that there exist nonnegative integers $x_{u}$ such that $\sum_{u \in V(G)} x_{u} \delta_{u}^{+}(G)=\sum_{u \in V(G)} x_{u} \delta_{u}^{-}(G)=\lambda(v-1)$ holds. A celebrated result of Wilson [36] proves these two necessary conditions also to be sufficient for the existence of a $(v, G, 1)$ digraph design, whenever $v$ is large enough. In a recent paper, Lamken and Wilson [27] extended this result to digraph families $\mathcal{G}$ and arbitrary $\lambda$.

Constructions for block designs, in particular, and digraph designs, in general, usually permit repeated blocks. In many cases, naturally, we are more interested in designs without repeated blocks. These designs are said to be simple. For details on the repeated block problem in design theory, the interested reader is referred to [7, 9]. Simple block designs have been studied e.g. in [13, 28, 32].

Many papers focus on designs satisfying somewhat stronger conditions. Bennett and Mendelsohn [4] asked for pure digraph designs where no two blocks have the same vertex set. It is easy to see that for $\lambda=1$, every digraph design is simple, but not necessarily pure.

Gronau and Mullin [16] asked for supersimple designs, i.e. block designs where any two distinct blocks have at most two elements in common. A number of papers is devoted to this question, cf. [1, 20, 25, 26].

A graph design is called suborthogonal if any two distinct blocks share at most one edge. Recent results on this issue can be found e.g. in $[10,33]$. Suborthogonal 
digraph designs with $\lambda=1$ are studied in [18]. Though not in the scope of this paper, it should be mentioned that a considerable number of papers deals with graph designs where any two distinct blocks share exactly one edge. For $\lambda=2$ these designs are known as orthogonal double covers. For a survey on this topic, cf. [2, 17].

In this paper, we go one step further and study digraph designs where any two blocks have at most two vertices in common. Following the terminology of $[4,16]$, we call these designs superpure. It is noteworthy, that this condition is in a certain sense strongest possible: A digraph design where any two distinct blocks share less than two vertices, will always be a block design with $\lambda=1$.

Our objective is to generalize the result of Lamken and Wilson [27] to superpure digraph designs. We claim that the well-known necessary conditions even ensure the existence of a superpure $(v, \mathcal{G}, \lambda)$ digraph design for sufficiently large $v$.

\section{Preliminaries}

In this section we rephrase some terminology and assemble basic results to be used in the sequel. In particular, we introduce arc colorings. Following [27], our results will be presented using colored digraphs. This concept is motivated by a variety of applications as pointed out in [27]. Further examples are contained in Section 8 below.

Throughout, $C$ denotes a fixed $r$-element set of colors. A digraph $G$ is said to be $r$-colored if its arcs are assigned colors from $C$. In this paper, we are exclusively concerned with arc colorings. Let $G_{c}$ denote the subdigraph of $G$ containing all arcs colored with color $c \in C$, and call it a page of $G$. It is convenient to use the notion $\underline{G}$ to refer to an $r$-colored digraph. Here, $\underline{G}$ may be viewed as an $r$-element vector whose components are the pages $G_{c}, c \in C$.

Given a family $\mathcal{G}$ of $r$-colored digraphs, let $\mathcal{G}_{c}$ denote the family of pages $G_{c}$ where $\underline{G}$ ranges over all digraphs in $\mathcal{G}$. Now, we are ready to extend the notion of a digraph design to colored digraphs. Let $\underline{H}$ be an $r$-colored digraph, $\mathcal{G}$ be a family of $r$ colored digraphs, and $\underline{\lambda}$ be an $r$-element vector of positive integers. A family $\mathcal{F}$ of subdigraphs of $\underline{H}$ is a $(\mathcal{G}, \underline{\lambda})$ decomposition of $\underline{H}$ if each $\mathcal{F}_{c}, c \in C$, forms a $\left(\mathcal{G}_{c}, \lambda_{c}\right)$ decomposition of the page $H_{c}$. It is customary, to call the members of $\mathcal{F}$ blocks.

Again, a $(\mathcal{G}, \underline{\lambda})$ decomposition of the complete $r$-colored digraph $\underline{D}_{v}$ with $v$ vertices is called a $(v, \mathcal{G}, \underline{\lambda})$ digraph design. If $\mathcal{G}$ consists of a single digraph $\underline{G}$, we shall briefly write $(v, \underline{G}, \underline{\lambda})$.

The definition of (multicolored) digraph designs immediately implies necessary conditions for their existence. In order to express this more succinctly, we introduce some technical notions. Let $\underline{\epsilon}(\underline{G})$ be the $r$-element vector containing the arc numbers of the pages of $\underline{G}$, and for every vertex $u$ in $\underline{G}$, let $\underline{\delta}_{u}(\underline{G})$ be the $2 r$-element vector whose components are the in- and outdegrees of $u$ with respect to the pages of $\underline{G}$. Henceforth, we call $\underline{\epsilon}(\underline{G})$ and $\underline{\delta}_{u}(\underline{G})$ the arc vector and the degree vector of $u$, respectively. By $\mathcal{B}(\mathcal{G})$ we denote the set of all arc vectors $\underline{\epsilon}(\underline{G})$ with $\underline{G}$ in $\mathcal{G}$, and by $\mathcal{A}(\mathcal{G})$ the set of all degree vectors with $\underline{G}$ in $\mathcal{G}$ and $u$ vertex in $\underline{G}$. 
Given two $r$-element vectors $\underline{x}$ and $\underline{y}$, let $\underline{x} \circ \underline{y}$ denote their Hadamard product, i.e. the $r$-element vector $\underline{z}$ with components $z_{c}=x_{c} y_{c}, c \in C$. Further, for a set $\mathcal{M}$ of $r$-element vectors, let $\mathcal{M}^{+}$denote its finite non-negative integral closure, i.e. the set of all finite non-negative integral linear combinations of vectors in $\mathcal{M}$.

Lemma 1. An r-colored digraph $\underline{H}$ admits a $(\mathcal{G}, \underline{\lambda})$ decomposition only if $\underline{\delta}_{u}(\underline{H}) \circ$ $\left(\frac{\lambda}{\underline{\lambda}}\right) \in \mathcal{A}^{+}(\mathcal{G})$, for every vertex $u$ in $\underline{H}$, and $\underline{\epsilon}(\underline{H}) \circ \underline{\lambda} \in \mathcal{B}^{+}(\mathcal{G})$ hold.

Proof. Let $\mathcal{F}$ be a $(\mathcal{G}, \underline{\lambda})$ decomposition of $\underline{H}$. Obviously, $\underline{\epsilon}(\underline{H}) \circ \underline{\lambda}$ equals the sum of the arc vectors $\underline{\epsilon}(\underline{F})$ when $\underline{F}$ ranges over $\mathcal{F}$. However, every member of $\mathcal{F}$ is a copy of a digraph in $\mathcal{G}$. Hence, $\mathcal{B}^{+}(\mathcal{G})$ contains $\underline{\epsilon}(\underline{H}) \circ \underline{\lambda}$. A similar argument proves the claim for $\mathcal{A}^{+}(\mathcal{G})$.

For the sake of simplicity, let $A(\mathcal{G}, \underline{\lambda})$ be the set of all positive integers $z$ with $z\left(\frac{\lambda}{\underline{\lambda}}\right) \in \mathcal{A}^{+}(\mathcal{G})$, and $B(\mathcal{G}, \underline{\lambda})$ be the set of all positive integers $z$ with $z \underline{\lambda} \in \mathcal{B}^{+}(\mathcal{G})$. For every digraph $\underline{G}$ in $\mathcal{G}$ the vector $\left(\frac{\underline{\epsilon}(\underline{G})}{\underline{G}}\right)$ is a non-negative integral linear combination of the degree vectors $\underline{\delta}_{u}(\underline{G})$, and thus belongs to $\mathcal{A}^{+}(\mathcal{G})$. Consequently, we have $\left(\frac{x}{x}\right) \in \mathcal{A}^{+}(\mathcal{G})$ for every vector $\underline{x} \in \mathcal{B}^{+}(\mathcal{G})$, which proves $A(\mathcal{G}, \underline{\lambda})$ to be a superset of $\bar{B}(\mathcal{G}, \underline{\lambda})$.

A pair $(\mathcal{G}, \underline{\lambda})$ is said to be admissible, whenever $B(\mathcal{G}, \underline{\lambda})$ is non-empty. This forces $A(\mathcal{G}, \underline{\lambda})$ to be non-empty, too, since $B(\mathcal{G}, \underline{\lambda})$ is a subset of $A(\mathcal{G}, \underline{\lambda})$. Evidently, digraph designs do only exist for admissible pairs $(\mathcal{G}, \underline{\lambda})$, as $(v-1) \in A(\mathcal{G}, \underline{\lambda})$ and $v(v-1) \in$ $B(\mathcal{G}, \underline{\lambda})$ must be satisfied due to Lemma 1 .

For admissible $(\mathcal{G}, \underline{\lambda})$, put $\alpha(\mathcal{G}, \underline{\lambda})=\operatorname{gcd} A(\mathcal{G}, \underline{\lambda})$ and $\beta(\mathcal{G}, \underline{\lambda})=\operatorname{gcd} B(\mathcal{G}, \underline{\lambda})$. This gives us the necessary conditions

$$
\begin{aligned}
v-1 & \equiv 0 \bmod \alpha(\mathcal{G}, \underline{\lambda}), \\
v(v-1) & \equiv 0 \bmod \beta(\mathcal{G}, \underline{\lambda})
\end{aligned}
$$

for the existence of a $(v, \mathcal{G}, \underline{\lambda})$ digraph design. By the work of Lamken and Wilson [27], these conditions happen to be sufficient for large $v$.

Theorem 2 (Lamken and Wilson). For every admissible $(\mathcal{G}, \underline{\lambda})$ and almost all positive integers $v$ satisfying the necessary conditions (1) and (2) there exists a $(v, \mathcal{G}, \underline{\lambda})$ digraph design.

Now we turn to the object of this paper. We call a $(v, \mathcal{G}, \underline{\lambda})$ digraph design superpure whenever any two distinct blocks share at most two vertices. Our objective is to prove the following stronger version of the preceding result.

Theorem 3. For every admissible $(\mathcal{G}, \underline{\lambda})$ and almost all positive integers $v$ satisfying the necessary conditions (1) and (2) there exists a superpure $(v, \mathcal{G}, \underline{\lambda})$ digraph design.

The remainder of the paper is organized as follows. In Sections 3 and 4, we provide constructions for $(v, \underline{G}, \underline{1})$ digraph designs where $\underline{1}$ is the vector with all elements equal to 1. Later on, these results are generalized in Section 5 to arbitrary pairs $(\mathcal{G}, \underline{\lambda})$. In Section 6 , we use the well-known concept of eventually periodic sets to 
prove our main result. Section 7 is devoted to digraph designs that split into smaller ones. Finally, in Section 6 we shall discuss some applications of the issue we are going to tackle in this paper.

\section{Constructions in finite FiEldS}

Throughout this section, let $\underline{G}$ denote an $r$-colored digraph with $n$ vertices and arc vector $\underline{\epsilon}(\underline{G})=\epsilon \underline{1}$ for some positive integer $\epsilon$. Suppose $\underline{G}$ has the vertex set $V(\underline{G})=\{1, \ldots, n\}$, and let $E(\underline{G})$ denote the arc set of $\underline{G}$.

We begin with some technical details, which help to prove the main result of this section, namely Lemma 7 . For a prime power $q \equiv 1 \bmod \epsilon$, we consider the finite field $\mathrm{GF}(q)$ of order $q$ with elements $0,1, \ldots, q-1$. Let $\Gamma$ be the cyclic multiplicative group containing all non-zero elements of $\mathrm{GF}(q)$, and let $T$ denote its unique subgroup of index $\epsilon$. If $g$ is a generator of $\Gamma$, then $T$ is obviously generated by $g^{\epsilon}$. For every integer $i$, the set $T_{i}=g^{i} T$ forms a coset of $\Gamma$ modulo $T$. Here, $T_{i}$ and $T_{j}$ denote the same coset whenever $i \equiv j \bmod \epsilon$ holds. The cosets themselves form again a cyclic group $\Gamma / T$, known as the factor group of $\Gamma$ modulo $T$. The order of $\Gamma / T$ is just $\epsilon$.

A major tool for our future investigation is a result from [19].

Lemma 4. Let $m, s$ be non-negative integers. For almost all prime powers $q \equiv$ $1 \bmod \epsilon$, for all m-element sets $X \subseteq G F(q)$, for all s-element sets $Z \subseteq G F(q)$ and for all maps $\theta: X \rightarrow \Gamma / T$ there exists an element $y \in G F(q)$ satisfying

$$
\begin{gathered}
y \notin Z, \\
y-x \in \theta(x), \text { for every } x \in X .
\end{gathered}
$$

To continue with, we define for every subset $M$ of $\mathrm{GF}(q)$ the sets

$$
\begin{aligned}
Z^{\prime}(M)= & \left\{y \in \operatorname{GF}(q): y=\left(b^{\prime} a+c^{\prime} b-a^{\prime} b-c^{\prime} a\right)\left(b^{\prime}-a^{\prime}\right)^{-1},\right. \\
& \text { where } \left.\{a, b\} \text { is a 2-element, and }\left\{a^{\prime}, b^{\prime}, c^{\prime}\right\} \text { is a 3-element subset of } M\right\}, \\
Z^{\prime \prime}(M)= & \left\{y \in \operatorname{GF}(q): y^{2}-\left(b+b^{\prime}\right) y+b^{\prime} a+c^{\prime} b-c^{\prime} a\right)=0, \\
& \text { where } \left.\{a, b\} \text { and }\left\{b^{\prime}, c^{\prime}\right\} \text { are 2-element subsets of } M\right\}, \\
Z^{\prime \prime \prime}(M)= & \left\{y \in \operatorname{GF}(q): y=\left(b^{\prime} a-a^{\prime} b\right)\left(-b-a^{\prime}+a+b^{\prime}\right)^{-1},\right. \\
& \text { where } \left.\{a, b\} \text { and }\left\{a^{\prime}, b^{\prime}\right\} \text { are 2-element subsets of } M\right\},
\end{aligned}
$$

and denote their union together with $\{0\}$ by $Z(M)$, which is going to play the role of the forbidden set $Z$ in Lemma 4 . Clearly, for an $m$-element set $M$, the set $Z(M)$ is of size at most $m^{5}+3 m^{4}+1$.

The next result was obtained in [27], and is recorded here for further reference as follows. Given a set $M$, a map - : $M \rightarrow M$ is a fixpoint-free involution, whenever $-x \neq x$ and $-(-x)=x$ holds for every $x \in M$. 
Lemma 5 (Lamken and Wilson). Let $\epsilon$ be even, $M$ be a set of size $\epsilon$, and - : $M \rightarrow M$ be a fixpoint-free involution. There exists a map $\phi: E(\underline{G}) \rightarrow M$ satisfying

$$
\begin{gathered}
\phi(e) \neq \phi\left(e^{\prime}\right) \text {, whenever } e \text { and } e^{\prime} \text { are distinct arcs of the same color, } \\
\phi(e)=-\phi\left(e^{\prime}\right) \text {, whenever the arcs } e \text { and } e^{\prime} \text { form a digon in } \underline{G} .
\end{gathered}
$$

Suppose, we restrict ourselves to odd $q$, and odd order of $T$. Then the field element -1 does not belong to $T$. Thus, mapping $T_{i}$ to $-T_{i}$ yields a fixpoint-free involution defined on the factor group $\Gamma / T$. For this particular involution, we make the following observation.

Lemma 6. Let $\epsilon$ be even. For almost all prime powers $q \equiv \epsilon+1 \bmod 2 \epsilon$, and every map $\phi: E(\underline{G}) \rightarrow \Gamma / T$ satisfying (6), there exist mutually distinct elements $x_{1}, \ldots, x_{n} \in G F(q)$ such that

$$
\begin{aligned}
x_{k} \notin Z\left(\left\{x_{1}, \ldots, x_{k-1}\right\}\right), \text { for } k=1, \ldots, n, & \\
& x_{j}-x_{i} \in \phi(e), \text { for every arc } e=(i, j) \text { in } \underline{G} .
\end{aligned}
$$

Proof. We use induction on $k$ where $k$ runs from 1 to $n$. The claim is an immediate consequence of Lemma 4 . To see this, we put $X=\left\{x_{1}, \ldots, x_{k-1}\right\}, Z=Z(X)$ and

$$
\theta\left(x_{i}\right)= \begin{cases}\phi(e), & \text { if } e=(i, k) \text { is an } \operatorname{arc} \text { in } \underline{G}, \\ -\phi(e), & \text { if } e=(k, i) \text { is an } \operatorname{arc~in} \underline{G}, \\ T, & \text { otherwise. }\end{cases}
$$

Due to condition (6), this definition makes sense. Moreover, $X$ is of size $(k-1)$, and $Z$ of size at most $(k-1)^{5}+3(k-1)^{4}+1$. Thus, Lemma 4 ensures the existence of a suitable element $x_{k}$.

We are now in the position to state the main result of this section.

Lemma 7. Let $\epsilon$ be even. For almost all prime powers $q \equiv \epsilon+1 \bmod 2 \epsilon$, there exists a superpure $(q, \underline{G}, \underline{1})$ digraph design.

Proof. By virtue of Lemma 5, there exists a map $\phi: E(\underline{G}) \rightarrow \Gamma / T$ satisfying conditions (5) and (6) for every prime power $q \equiv \epsilon+1 \bmod 2 \epsilon$. For almost each of these values, we find mutually distinct elements $x_{1}, \ldots, x_{n}$ in $\operatorname{GF}(q)$ such that $(7)$ and (8) hold.

Suppose, that $\underline{G}$ has vertex set $V(\underline{G})=\{1, \ldots, n\}$. For every $t \in T$, and each element $u$ of $\operatorname{GF}(q)$, we construct a digraph $\underline{F}(t, u)$ as the copy of $\underline{G}$ under the map $i \rightarrow t x_{i}+u$. By virtue of the conditions (5) and (8), the family $\mathcal{F}$ containing all these digraphs forms a $(q, \underline{G}, \underline{1})$ digraph design, cf. [8, 27].

It remains to check that $\mathcal{F}$ is superpure. Assume, there are two different blocks $\underline{F}(t, u)$ and $\underline{F}\left(t^{\prime}, u^{\prime}\right)$ in $\mathcal{F}$ sharing more than two vertices. Then there must be 
3-element subsets $\{i, j, k\}$ and $\left\{i^{\prime}, j^{\prime}, k^{\prime}\right\}$ of the vertex set $V(\underline{G})$ such that

$$
\begin{aligned}
& t x_{i}+u=t^{\prime} x_{i^{\prime}}+u^{\prime} \\
& t x_{j}+u=t^{\prime} x_{j^{\prime}}+u^{\prime} \\
& t x_{k}+u=t^{\prime} x_{k^{\prime}}+u^{\prime}
\end{aligned}
$$

hold. Thus $t, t^{\prime}$ and $u-u^{\prime}$ yield a non-trivial solution to the system of linear equations

$$
\left(\begin{array}{lll}
x_{i} & -x_{i^{\prime}} & 1 \\
x_{j} & -x_{j^{\prime}} & 1 \\
x_{k} & -x_{k^{\prime}} & 1
\end{array}\right)\left(\begin{array}{c}
t \\
t^{\prime} \\
u-u^{\prime}
\end{array}\right)=\left(\begin{array}{l}
0 \\
0 \\
0
\end{array}\right) .
$$

Consequently, the determinant $-x_{j^{\prime}} x_{i}-x_{k^{\prime}} x_{j}-x_{i^{\prime}} x_{k}+x_{k^{\prime}} x_{i}+x_{i^{\prime}} x_{j}+x_{j^{\prime}} x_{k}$ of the coefficient matrix equals 0 . Suppose, $k$ is maximal among the integers $i, j, k, i^{\prime}, j^{\prime}, k^{\prime}$. A straightforward calculation confirms

$$
x_{k}=\left(x_{j^{\prime}} x_{i}+x_{k^{\prime}} x_{j}-x_{i^{\prime}} x_{j}-x_{k^{\prime}} x_{i}\right)\left(x_{j^{\prime}}-x_{i^{\prime}}\right)^{-1} .
$$

If $k$ is different from $i^{\prime}, j^{\prime}, k^{\prime}$, this gives us $x_{k} \in Z^{\prime}\left(\left\{x_{1}, \ldots, x_{k-1}\right\}\right)$, which contradicts condition (7). Otherwise, let $k$ belong to $\left\{i^{\prime}, j^{\prime}, k^{\prime}\right\}$. For $k=i^{\prime}$, we obtain

$$
x_{k}^{2}-\left(x_{j}+x_{j^{\prime}}\right) x_{k}+x_{j^{\prime}} x_{i}+x_{k^{\prime}} x_{j}-x_{k^{\prime}} x_{i}=0 .
$$

Therefore, $x_{k}$ lies in $Z^{\prime \prime}\left(\left\{x_{1}, \ldots, x_{k-1}\right\}\right)$, which contradicts condition (7), too. A similar argument applies for $k=j^{\prime}$. Finally, let $k=k^{\prime}$. This implies

$$
x_{k}\left(-x_{j}-x_{i^{\prime}}+x_{i}+x_{j^{\prime}}\right)=x_{j^{\prime}} x_{i}-x_{i^{\prime}} x_{j} .
$$

When inspecting this relation, we obtain either $-x_{j}-x_{i^{\prime}}+x_{i}+x_{j^{\prime}} \neq 0$, or $x_{i}-x_{j}=$ $x_{i^{\prime}}-x_{j^{\prime}}$ together with $x_{j^{\prime}} x_{i}=x_{i^{\prime}} x_{j}$. The first alternative would amount to saying $x_{k} \in Z^{\prime \prime \prime}\left(\left\{x_{1}, \ldots, x_{k-1}\right\}\right)$ which contradicts $(7)$. Due to the second alternative, we infer $i=i^{\prime}$ and $j=j^{\prime}$, and consequently $t=t^{\prime}$ as well as $u=u^{\prime}$. But this is a contradiction to $\underline{F}(t, u)$ and $\underline{F}\left(t^{\prime}, u^{\prime}\right)$ being different.

As a result of the above discussion, we have shown $\mathcal{F}$ to be superpure, which concludes the proof.

\section{PBD-CLOSURE AND PERIODS}

To continue our study, we need some standard terminology from design theory. Let $K$ be a subset of $\mathbb{N}$, the set of all positive integers. A pairwise balanced design $\operatorname{PBD}(v, K)$ is a family of subsets of a $v$-element set, such that every pair of distinct elements occurs in exactly one subset, and the size of every subset is in $K$. Clearly, a $\operatorname{PBD}(v, K)$ corresponds to a $(v, \mathcal{D}, 1)$ digraph design where $\mathcal{D}$ is the family of complete digraphs $D_{k}$ with $k$ running over $K$. A set $K$ is $P B D$-closed if the existence of a $\operatorname{PBD}(v, K)$ immediately implies $v \in K$.

Lemma 8. Let $\mathcal{G}$ be a family of $r$-colored digraphs, and $\underline{\lambda}$ an r-element vector of positive integers. For admissible pairs $(\mathcal{G}, \underline{\lambda})$, the set $S(\mathcal{G}, \underline{\lambda})=\{v \in \mathbb{N}$ : there exists a superpure $(v, \mathcal{G}, \underline{\lambda})$ digraph design $\}$ is PBD-closed. 
Proof. Suppose, $v$ is an integer admitting a $\operatorname{PBD}(v, K)$ with $K=S(\mathcal{G}, \underline{\lambda})$. This yields the existence of a $(v, \mathcal{D}, 1)$ digraph design where $\mathcal{D}$ contains the complete digraph $D_{k}$ for every $k \in S(\mathcal{G}, \underline{\lambda})$. Moreover, we have a superpure $(\mathcal{G}, \underline{\lambda})$ decomposition of any of these complete digraphs $D_{k}$. Replacing each of the blocks $D$ in the $(v, \mathcal{D}, 1)$ digraph design by the corresponding $(\mathcal{G}, \underline{\lambda})$ decomposition $\mathcal{F}_{D}$ of $D$ provides a $(v, \mathcal{G}, \underline{\lambda})$ digraph design $\mathcal{F}$.

Next, consider two different digraphs from $\mathcal{F}$. If they belong to the same family $\mathcal{F}_{D}$, they share at most two vertices as $\mathcal{F}_{D}$ happens to be superpure. Conversely, if the digraphs are from different families $\mathcal{F}_{D}$, there vertex sets are subsets of different blocks of the underlying $\operatorname{PBD}(v, K)$. Hence, they have at most one vertex in common. This proves $\mathcal{F}$ to be superpure.

Given integers $\pi \geq 1$ and $u$, put $\mathbb{N}_{u(\pi)}=\{v \in \mathbb{N}: v \equiv u \bmod \pi\}$. For a set $K$ of positive integers, every non-empty subset $K \cap \mathbb{N}_{u(\pi)}$ shall be called a $\pi$-fibre of $K$. Furthermore, $K$ is eventually periodic with period $\pi$ if each of its $\pi$-fibres $K \cap \mathbb{N}_{u(\pi)}$ contains almost all integers from $\mathbb{N}_{u(\pi)}$. If $\pi$ is a period of $K$, then all multiples of $\pi$ are periods, too. In fact, the periods of an eventually periodic set $K$ consist of the multiples of a primitive period, that is, the greatest common divisor of all periods of $K$. Put $\alpha(K)=\operatorname{gcd}\{k-1: k \in K\}$ and $\beta(K)=\operatorname{gcd}\{k(k-1): k \in K\}$. Due to Wilson [35], every non-empty PBD-closed set $K \neq\{1\}$ is eventually periodic. Its primitive period is $\frac{1}{2} \beta(K)$ if $\alpha(K)$ and $\frac{1}{2} \beta(K)$ are odd, or $\beta(K)$ otherwise. Below we make use of the following simple observation:

Lemma 9. Let $K$ and $L$ be non-empty $P B D$-closed sets with $\{1\} \neq K \subseteq L$. Every period of $K$ is a period of $L$, too.

Proof. Clearly, $\alpha(K)$ and $\beta(K)$ are multiples of $\alpha(L)$ and $\beta(L)$, respectively. If $\beta(K)$ is the primitive period of $K$, this immediately yields the claim. If $\frac{1}{2} \beta(K)$ is the primitive period of $K$, then $\alpha(K)$ and $\frac{1}{2} \beta(K)$ are odd. Consequently $\alpha(L)$ and $\frac{1}{2} \beta(L)$ are odd, too, which again implies the claim.

As in Section 3, let $\underline{G}$ be an $r$-colored digraph with arc vector $\underline{\epsilon}(\underline{G})=\epsilon \underline{1}$ for some positive integer $\epsilon$. By virtue of Lemma 8 , the set $S(\underline{G}, \underline{1})$ is eventually periodic. It would be interesting to infer a suitable period for this set. Dirichlet's theorem on primes in arithmetic progressions turns out to be a useful tool for that.

Theorem 10 (Dirichlet). Let $m$ and $u$ be positive integers with $\operatorname{gcd}\{m, u\}=1$. Then there exist infinitely many primes $p \equiv u \bmod m$.

Applying our observations from the preceding section, we conclude the following result.

Lemma 11. The set $S(\underline{G}, \underline{1})$ has period

$$
\pi(\underline{G})= \begin{cases}\epsilon, & \text { if } \epsilon \text { is even } \\ 2 \epsilon, & \text { otherwise. }\end{cases}
$$


Proof. It suffices to determine $\beta(S(\underline{G}, \underline{1}))$ or at least some multiple of this value, which will always be a period of $S(\underline{G}, \underline{1})$, too. First, let $\epsilon$ be even. By Lemma 7 , $S(\underline{G}, \underline{1})$ contains almost all primes $q \equiv \epsilon+1 \bmod 2 \epsilon$.

By virtue of Dirichlet's theorem, $S(\underline{G}, \underline{1})$ contains a prime

$$
p \equiv 2 \epsilon^{2}+\epsilon+1 \bmod 2 \epsilon(\epsilon+1),
$$

say $p=2 \epsilon(\epsilon+1) t+2 \epsilon^{2}+\epsilon+1=\epsilon t^{\prime}+1$ for suitable integers $t$ and $t^{\prime}$. Note that $\operatorname{gcd}\left\{\epsilon+1, t^{\prime}\right\}=\operatorname{gcd}\{\epsilon+1, \epsilon\}=1$ holds.

The same argument shows, that $S(\underline{G}, \underline{1})$ contains a second prime $p^{\prime}>p$ with

$$
p^{\prime} \equiv \epsilon+1 \bmod 2 \epsilon p t^{\prime},
$$

say $p^{\prime}=2 \epsilon p t^{\prime} s+\epsilon+1$ for some integer $s$. Now, we have

$$
\operatorname{gcd}\left\{p(p-1), p^{\prime}\left(p^{\prime}-1\right)\right\}=\operatorname{gcd}\left\{p(p-1), p^{\prime}-1\right\}=\epsilon \operatorname{gcd}\left\{p t^{\prime}, 2 p t^{\prime} s+1\right\}=\epsilon .
$$

Hence, $\epsilon$ is a multiple of $\beta(S(\underline{G}, \underline{1}))$, i.e. a period of $S(\underline{G}, \underline{1})$.

For the case of odd $\epsilon$, let $\underline{G}^{\prime}$ denote the union of two disjoint copies of $\underline{G}$. Obviously, $S\left(\underline{G}^{\prime}, \underline{1}\right) \subseteq S(\underline{G}, \underline{1})$ holds. Moreover, we have $\underline{\epsilon}\left(\underline{G}^{\prime}\right)=2 \underline{\epsilon}(\underline{G})=2 \epsilon \underline{1}$, which gives us $2 \epsilon$ as a period for $S\left(\underline{G}^{\prime}, \underline{1}\right)$. By Lemma $9, S(\underline{G}, \underline{1})$ has period $2 \epsilon$, too.

Corollary 12. There exists a superpure $(v, \underline{G}, \underline{1})$ digraph design for almost every integer $v \equiv 1 \bmod \pi(\underline{G})$.

Proof. Let $\epsilon$ be even. By Lemma 7 and Dirichlet's theorem on primes in arithmetic progressions, $S(\underline{G}, \underline{1})$ contains a prime $q \equiv 1 \bmod \epsilon$, which implies the claim. For odd $\epsilon$, we again consider the disjoint union $\underline{G}^{\prime}$ of two copies of $\underline{G}$. By the same argument, $S\left(\underline{G}^{\prime}, \underline{1}\right)$ contains a prime $q \equiv 1 \bmod 2 \epsilon$. This observation concludes the proof, because $S\left(\underline{G}^{\prime}, \underline{1}\right)$ is a subset of $S(\underline{G}, \underline{1})$.

\section{Assemblies AND REFinEmEnts}

In the sequel we shall generalize the results obtained in the preceding section to arbitrary $(\mathcal{G}, \underline{\lambda})$. Throughout, let $\underline{G}$ be an $r$-colored digraph, $\mathcal{G}$ be a family of $r$ colored digraphs, $\underline{\lambda}$ be an $r$-element vector of positive integers. Moreover, we assume $(\mathcal{G}, \underline{\lambda})$ to be admissible.

A pair $\left(\underline{G}^{\prime}, \underline{\lambda}\right)$ is an assembly of $(\mathcal{G}, \underline{\lambda})$ if the digraph $\underline{G}^{\prime}$ is a disjoint union of copies of digraphs in $\mathcal{G}$ such that its arc vector $\underline{\epsilon}\left(\underline{G}^{\prime}\right)$ equals $\epsilon \underline{\lambda}$ for some positive integer $\epsilon$. It is trivial to check, that every admissible pair $(\mathcal{G}, \underline{\lambda})$ admits an assembly.

Lemma 13. Let $\left(\underline{G}^{\prime}, \underline{\lambda}\right)$ be an assembly of $(\mathcal{G}, \underline{\lambda})$. The existence of a superpure $(v, \underline{G}, \underline{\lambda})$ digraph design yields the existence of a superpure $(v, \mathcal{G}, \underline{\lambda})$ digraph design.

Proof. Let $\mathcal{F}^{\prime}$ be a superpure $\left(v, \underline{G}^{\prime}, \underline{\lambda}\right)$ digraph design. Every block $\underline{F}^{\prime}$ in $\mathcal{F}^{\prime}$ is a copy of $\underline{G}^{\prime}$, i.e. the disjoint union of copies of digraphs from $\mathcal{G}$. Denote the family of these copies by $\mathcal{F}_{\underline{F}^{\prime}}$. When replacing $\underline{F}^{\prime}$ by the family $\mathcal{F}_{\underline{F}^{\prime}}$, we obtain a $(v, \mathcal{G}, \underline{\lambda})$ digraph design $\mathcal{F}$. Moreover, any two blocks of $\mathcal{F}$ are vertex-disjoint if they belong 
to the same family $\mathcal{F}_{\underline{F}^{\prime}}$. Conversely, if they belong to different families $\mathcal{F}_{\underline{F}^{\prime}}$, they share at most two vertices since $\mathcal{F}^{\prime}$ was superpure.

As above, let $C$ denote the $r$-element set containing the colors assigned to the arcs of $\underline{G}$. Moreover, let $C^{\prime}$ be an $r^{\prime}$-element set of colors assigned to an $r^{\prime}$-colored digraph $\underline{G}^{\prime}$ with $r^{\prime}>r$, and let $\psi: C^{\prime} \rightarrow C$ be a map onto $C$. Then $\left(\underline{G}^{\prime}, \underline{\lambda}^{\prime}\right)$ is a refinement of a pair $(\underline{G}, \underline{\lambda})$ if $\underline{G}$ is obtained from $\underline{G}^{\prime}$ by assigning the color $\psi\left(c^{\prime}\right)$ to every arc of color $c^{\prime}$ in $\underline{G}^{\prime}$, and $\underline{\lambda}^{\prime}$ denotes an $r^{\prime}$-element vector of positive integers, such that $\underline{\lambda}$ has components $\lambda_{c}=\sum_{c^{\prime} \in C^{\prime}: \psi\left(c^{\prime}\right)=c} \lambda_{c^{\prime}}^{\prime}$ for every color $c \in C$. Due to this definition, $\underline{G}$ and $\underline{G}^{\prime}$ actually mean the same digraph, but with different colorings.

Lemma 14. Let $\left(\underline{G}^{\prime}, \underline{\lambda}^{\prime}\right)$ be a refinement of $(\underline{G}, \underline{\lambda})$. The existence of a superpure $\left(v, \underline{G}^{\prime}, \underline{\lambda}^{\prime}\right)$ digraph design yields the existence of a superpure $(v, \underline{G}, \underline{\lambda})$ digraph design.

Proof. Let $\mathcal{F}^{\prime}$ be a superpure $\left(v, \underline{G}^{\prime}, \underline{\lambda}^{\prime}\right)$ digraph design. When assigning color $\psi\left(c^{\prime}\right)$ to every arc of color $c^{\prime} \in C^{\prime}$ in the blocks of $\mathcal{F}^{\prime}$, we immediately obtain a $(v, \underline{G}, \underline{\lambda})$ digraph design $\mathcal{F}$. Furthermore, any two blocks in $\mathcal{F}^{\prime}$ share the same vertices as their images in $\mathcal{F}$. This forces $\mathcal{F}$ to be superpure, too.

In order to apply the results from the preceding section, we are interested in those pairs $(\underline{G}, \underline{\lambda})$ which admit a refinement $\left(\underline{G}^{\prime}, \underline{1}\right)$.

Lemma 15. There exists a digraph $\underline{G}^{\prime}$ such that $\underline{\epsilon}\left(\underline{G}^{\prime}\right)=\epsilon \underline{1}$ holds for some positive integer $\epsilon$ and $\left(\underline{G}^{\prime}, \underline{1}\right)$ is a refinement of $(\underline{G}, \underline{\lambda})$ if and only if $\underline{G}$ has arc vector $\underline{\epsilon}(\underline{G})=$ $\epsilon \underline{\lambda}$.

Proof. Let $G^{\prime}$ be an $r^{\prime}$-colored digraph satisfying the assumptions of the lemma, and as above let $C^{\prime}$ contain the colors assigned to the arcs of $\underline{G}^{\prime}$. Since $\left(\underline{G}^{\prime}, \underline{1}\right)$ is a refinement of $(\underline{G}, \underline{\lambda})$, we have $\lambda_{c}=\left|\left\{c^{\prime} \in C^{\prime}: \psi\left(c^{\prime}\right)=c\right\}\right|$ for every color $c \in C$. On the other hand, the page $G_{c}$ of color $c \in C$ in the digraph $\underline{G}$ has just $\epsilon\left(G_{c}\right)=\sum_{c^{\prime} \in C^{\prime}: \psi\left(c^{\prime}\right)=c} \epsilon\left(G_{c^{\prime}}^{\prime}\right)$ arcs. This implies $\epsilon\left(G_{c}\right)=\epsilon \lambda_{c}$, and thus the claimed result.

Conversely, let the arc vector of $\underline{G}$ be equal to $\epsilon \underline{\lambda}$ for some positive integer $\epsilon$. Further, let $C^{\prime}$ be an $r^{\prime}$-element set of colors, and $\psi: C^{\prime} \rightarrow C$ a map such that there are exactly $\lambda_{c}$ elements in $C^{\prime}$ mapped to every color $c$. For each $c^{\prime} \in C^{\prime}$ choose precisely $\epsilon$ arcs of page $G_{c}$ where $c=\psi\left(c^{\prime}\right)$, and assign them the new color $c^{\prime}$. The resultant digraph $\underline{G}^{\prime}$ is $r^{\prime}$-colored and has arc vector $\underline{\epsilon}\left(\underline{G}^{\prime}\right)=\epsilon \underline{1}$. Thus, it is easy to check, that $\left(\underline{G^{\prime}}, \underline{1}\right)$ forms an assembly of $(\underline{G}, \underline{\lambda})$.

This observation enables us to determine periods of $S(\mathcal{G}, \underline{\lambda})$ for every admissible pair $(\mathcal{G}, \underline{\lambda})$. As pointed out earlier, there exists an assembly $(\underline{G}, \underline{\lambda})$ of $(\mathcal{G}, \underline{\lambda})$, and by Lemma 15, this assembly admits a refinement $\left(\underline{G}^{\prime}, \underline{1}\right)$. Applying Lemma 13 and 14, we obtain $S\left(\underline{G}^{\prime}, \underline{1}\right) \subseteq S(\underline{G}, \underline{\lambda}) \subseteq S(\mathcal{G}, \underline{\lambda})$. By Lemma 9 , every period of $S\left(\underline{G}^{\prime}, \underline{1}\right)$ is a period of $S(\mathcal{G}, \underline{\lambda})$, too. 


\section{BLOWING-UP DIGRAPH DESIGNS}

In this section, we finish the proof of Theorem 3. Throughout, let $\mathcal{G}$ be a family of $r$-colored digraphs, and $\underline{\lambda}$ an $r$-element vector of positive integers such that $(\mathcal{G}, \underline{\lambda})$ is admissible. As above, let $(\underline{G}, \underline{\lambda})$ be an assembly of $(\mathcal{G}, \underline{\lambda})$, and $\left(\underline{G}^{\prime}, \underline{1}\right)$ a refinement of $(\underline{G}, \underline{\lambda})$. By $\pi$ we denote the period of $S\left(\underline{G^{\prime}}, \underline{1}\right)$ given in Lemma 11. Our objective is to prove, that for every positive integer $u$ satisfying the necessary conditions (1) and (2) the set $S(\mathcal{G}, \underline{\lambda}) \cap \mathbb{N}_{u(\pi)}$ is non-empty, i.e. a $\pi$-fibre.

To do this, we shall use difference matrices. Given an abelian group $Y$ of order $g$ and a positive integer $h$, a $(g, h)$ difference matrix on $Y$ is an $h$-by- $g$ matrix $M=\left(m_{i j}\right)$ with entries from $Y$, such that for all $1 \leq k<l \leq h$, and all $1 \leq j<j^{\prime} \leq g$ the differences $m_{k j}-m_{l j}$ and $m_{k j^{\prime}}-m_{l j^{\prime}}$ are different. For every prime power $q$ and $h \leq q$, Drake [15] proved the existence of a $(q, h)$ difference matrix on the additive group of the finite field $\mathrm{GF}(q)$.

Lemma 16. Let $u$ be a positive integer satisfying (1) and (2). There is a positive integer $v \equiv u \bmod \pi$ such that there exists a superpure $(v, \mathcal{G}, \underline{\lambda})$ digraph design.

Proof. Let $D(\mathcal{G}, \underline{\lambda})$ be the set of all positive integers $v$ admitting a $(v, \mathcal{G}, \underline{\lambda})$ digraph design. This set is known to be eventually periodic, as shown in [27]. Since $S(\mathcal{G}, \underline{\lambda}) \subseteq$ $D(\mathcal{G}, \underline{\lambda})$ holds, $\pi$ is also a period of $D(\mathcal{G}, \underline{\lambda})$. Consequently, there exists a $(w, \mathcal{G}, \underline{\lambda})$ digraph design for some positive integer $w \equiv u \bmod \pi$.

Let $W=\{1, \ldots, w\}$, and suppose we are given such a $(w, \mathcal{G}, \underline{\lambda})$ digraph design $\mathcal{F}$ on the vertex set $V\left(\underline{D}_{w}\right)=W$ of the complete $r$-colored digraph $\underline{D}_{w}$. By $\left(\begin{array}{c}W \\ 2\end{array}\right)$, we denote the set of all 2-element subsets of $W$, which is of size $h=\left(\begin{array}{c}w \\ 2\end{array}\right)$.

By Dirichlet's theorem on primes in arithmetic progressions, there is a prime $q \equiv$ $1 \bmod \pi$, such that $q^{h} \in S(\mathcal{G}, \underline{\lambda})$ holds, and $q$ is larger than both, $h$ and the number of blocks in $\mathcal{F}$. By $X$, we denote the space of all $h$-element vectors with entries from $\operatorname{GF}(q)$. Clearly, $X$ is of size $s=q^{h}$, and $V=W \times X$ is of size $v=w s$. It is noteworthy, that $v=w s=w q^{h} \equiv w \equiv u \bmod \pi$, and we aim on constructing a superpure $(v, \mathcal{G}, \underline{\lambda})$ digraph design on the set $V$.

For the sake of simplicity, we suppose the components of any vector $\underline{x} \in X$ to be indexed by the members of $\left(\begin{array}{c}W \\ 2\end{array}\right)$, i.e. by 2-element subsets $K$ of $W$. Further, let $T$ be the $(h-1)$-dimensional subspace of $X$ containing all vectors $\underline{x}$ whose inner product with $\underline{1}$ equals 0 . It should be mentioned, that every vector different from $\underline{0}$ in $T$ has at least two non-zero components.

Let $\gamma$ be a generator of the multiplicative group of the finite field $\operatorname{GF}(q)$. For every vertex $i \in W$, we consider vectors $\underline{z}^{(i)}$ and $\underline{n}^{(i)} \in X$ with components

$$
z_{K}^{(i)}=\left\{\begin{array}{ll}
1 & \text { if } i \in K, \\
\gamma^{i} & \text { otherwise, }
\end{array} \text { and } n_{K}^{(i)}= \begin{cases}1 & \text { if } i=\max K \\
0 & \text { otherwise }\end{cases}\right.
$$

respectively. In addition, let $M$ be a $(q, h)$ difference matrix on $\operatorname{GF}(q)$. For every block $\underline{F}$ in $\mathcal{F}$, we fix a column $\underline{m}^{(F)}$ of the matrix $M$, such that no column is chosen twice. 
Now, we are ready to show how to obtain the blocks of the claimed $(v, \mathcal{G}, \underline{\lambda})$ digraph design. First, on each of the sets $\{i\} \times X$ with $i \in W$, we construct a superpure $(s, \mathcal{G}, \underline{\lambda})$ digraph design $\mathcal{F}_{i}$. This is possible since $s=q^{h}$ lies in $S(\mathcal{G}, \underline{\lambda})$.

In addition, for every block $\underline{F} \in \mathcal{F}$, every vector $\underline{x} \in X$, every vector $\underline{t} \in T$ and every element $a \in \mathrm{GF}(q)$, we construct a digraph $\underline{F}(\underline{x}, \underline{t}, a)$ with vertices from $V$ as the copy of $\underline{F}$ under the map $i \rightarrow(i, \underline{i})$ where

$$
\underline{i}=\underline{x}+\underline{t}^{\circ} \underline{z}^{(i)}+\left(a \underline{1}+\underline{m}^{(F)}\right) \circ \underline{n}^{(i)} .
$$

Hence, from every old block $\underline{F} \in \mathcal{F}$, we obtain exactly $s^{2}$ new blocks. The family of all these blocks shall be $\mathcal{F}_{0}$.

Firstly, we have to show that the union $\mathcal{F}^{\prime}$ of $\mathcal{F}_{0}$ and the families $\mathcal{F}_{i}$ with $i \in W$ forms a $(v, \mathcal{G}, \underline{\lambda})$ digraph design on $V$.

Let $((i, \underline{i}),(j, \underline{j}))$ be an arc of color $c$ in the $r$-colored complete digraph $\underline{D}_{v}$ on vertex set $V$. If $i=\bar{j}$, this arc appears exactly $\lambda_{c}$ times in $\mathcal{F}^{\prime}$ since $\mathcal{F}_{i}$ is an $(s, \mathcal{G}, \underline{\lambda})$ digraph design on $\{i\} \times X$. Conversely, let $i$ be different from $j$. In the original $(w, \mathcal{G}, \underline{\lambda})$ digraph design $\mathcal{F}$ there are precisely $\lambda_{c}$ blocks containing the arc $(i, j)$ of color $c$. Fix any of them, say $\underline{F}$. From $\underline{F}$ we obtained $s^{2}$ new blocks in $\mathcal{F}^{\prime}$.

Assume, there are two different copies $\underline{F}(\underline{x}, \underline{t}, a)$ and $\underline{F}\left(\underline{x}^{\prime}, \underline{t}^{\prime}, a^{\prime}\right)$ both containing the vertices $(i, \underline{i})$ and $(j, \underline{j})$. Without loss of generality, suppose $i<j$. This implies

$$
\begin{aligned}
& \underline{i}=\underline{x}+\underline{t} \circ \underline{z}^{(i)}+\left(a \underline{1}+\underline{m}^{(F)}\right) \circ \underline{n}^{(i)}=\underline{x}^{\prime}+\underline{t}^{\prime} \circ \underline{z}^{(i)}+\left(a^{\prime} \underline{1}+\underline{m}^{(\underline{F})}\right) \circ \underline{n}^{(i)} \\
& \underline{j}=\underline{x}+\underline{t}^{\prime} \circ \underline{z}^{(j)}+\left(a \underline{1}+\underline{m}^{(\underline{F)})}\right) \circ \underline{n}^{(j)}=\underline{x}^{\prime}+\underline{t}^{\prime} \circ \underline{z}^{(j)}+\left(a^{\prime} \underline{1}+\underline{m}^{(\underline{F)})} \circ \underline{n}^{(j)}\right.
\end{aligned}
$$

which gives us

$$
\begin{aligned}
\underline{j}-\underline{i} & =\underline{t} \circ\left(\underline{z}^{(j)}-\underline{z}^{(i)}\right)+\left(a \underline{1}+\underline{m} \underline{\underline{F}}^{(\underline{F})}\right) \circ\left(\underline{n}^{(j)}-\underline{n}^{(i)}\right) \\
& =\underline{t}^{\prime} \circ\left(\underline{z}^{(j)}-\underline{z}^{(i)}\right)+\left(a^{\prime} \underline{1}+\underline{m}^{(\underline{F})}\right) \circ\left(\underline{n}^{(j)}-\underline{n}^{(i)}\right) .
\end{aligned}
$$

Now we take a close look at the component with index $K=\{i, j\}$. We have $(\underline{j}-\underline{i})_{K}=a+m_{K}^{(F)}=a^{\prime}+m_{K}^{(F)}$, which implies $a=a^{\prime}$. From this, we derive $\left(\underline{t}^{\prime}-\underline{t}\right) \circ\left(\underline{z}^{(j)}-\underline{z}^{(i)}\right)=\underline{0}$. However, in $\left(\underline{z}^{(j)}-\underline{z}^{(i)}\right)$ all but one component (the one with index $K)$ are different from 0 . Hence, $\left(\underline{t}^{\prime}-\underline{t}\right) \in T$ contains at most one non-zero component. Consequently, this vector has to be $\underline{0}$ as pointed out earlier. We obtain $\underline{t}=\underline{t}^{\prime}$ and, moreover, $\underline{x}=\underline{x}^{\prime}$, which gives a contradiction to $\underline{F}(\underline{x}, \underline{t}, a)$ and $\underline{F}\left(\underline{x}^{\prime}, \underline{t}^{\prime}, a^{\prime}\right)$ being different. Therefore, any two different copies of a block $\underline{F}$ have at most one vertex in common.

Consider any vectors $\underline{i}, j \in X$ and any color $c \in C$. Recall that $\underline{F}$ is a fixed block from $\mathcal{F}$ containing the $\operatorname{arc}(i, j)$. The preceding observation means, in particular, that the arc $((i, \underline{i}),(j, \underline{j}))$ of color $c$ occurs in at most one copy of $\underline{F}$. Since the arc $(i, j)$ appears just in $\lambda_{c}$ blocks from $\mathcal{F}$, there are at most $\lambda_{c}$ blocks in $\mathcal{F}^{\prime}$ containing $((i, \underline{i}),(j, j))$. On the other hand, it should be noted that the digraphs in each of the families $\mathcal{F}_{i}$ altogether contain $s(s-1) \lambda_{c}$ arcs of color $c \in C$, and the digraphs in $\mathcal{F}_{0}$ altogether contain just $s^{2} w(w-1) \lambda_{c}$ arcs of color $c$. Thus, we obtain a total 
amount of

$$
w s(s-1) \lambda_{c}+s^{2} w(w-1) \lambda_{c}=w s(w s-1) \lambda_{c}=v(v-1) \lambda_{c}
$$

arcs, when counting all arcs of color $c \in C$ in any of the digraphs in $\mathcal{F}^{\prime}$. This is just the right number for a $(v, \mathcal{G}, \underline{\lambda})$ digraph design as desired. Hence, the arc $((i, \underline{i}),(j, \underline{j}))$ of color $c$ occurs in exactly $\lambda_{c}$ blocks in $\mathcal{F}^{\prime}$, i.e. $\mathcal{F}^{\prime}$ is a $(v, \mathcal{G}, \underline{\lambda})$ digraph design as desired.

It remains to check whether $\mathcal{F}^{\prime}$ is superpure as claimed. Since each of the families $\mathcal{F}_{i}$ is superpure, two different blocks from the same family $\mathcal{F}_{i}$ will never share more than two vertices. Due to our construction, a block from $\mathcal{F}_{i}$ and a block from $\mathcal{F}_{0}$ have at most one vertex in common. The same holds for two blocks from $\mathcal{F}_{0}$ which happen to be copies of the same old block $\underline{F} \in \mathcal{F}$, as seen above.

Suppose, we are given two different digraphs $\underline{F}(\underline{x}, \underline{t}, a)$ and $\underline{F}^{\prime}\left(\underline{x}^{\prime}, \underline{t}^{\prime}, a^{\prime}\right)$ from $\mathcal{F}_{0}$, which were obtained from different blocks $\underline{F}$ and $\underline{F}^{\prime}$ in $\mathcal{F}$. Assume, these digraphs share at least three vertices, say $(i, \underline{i}),(j, \underline{j})$ and $(k, \underline{k})$ with $i<j<k$. Again, we have

$$
\begin{aligned}
& \underline{i}=\underline{x}+\underline{t} \circ \underline{z}^{(i)}+\left(a \underline{1}+\underline{m}^{(\underline{F})}\right) \circ \underline{n}^{(i)}=\underline{x}^{\prime}+\underline{t}^{\prime} \circ \underline{z}^{(i)}+\left(a^{\prime} \underline{1}+\underline{m}^{\left(\underline{F}^{\prime}\right)}\right) \circ \underline{n}^{(i)} \\
& \underline{j}=\underline{x}+\underline{t} \circ \underline{z}^{(j)}+\left(a \underline{1}+\underline{m}^{(\underline{F})}\right) \circ \underline{n}^{(j)}=\underline{x}^{\prime}+\underline{t}^{\prime} \circ \underline{z}^{(j)}+\left(a^{\prime} \underline{1}+\underline{m}^{\left(\underline{F}^{\prime}\right)}\right) \circ \underline{n}^{(j)},
\end{aligned}
$$

as well as

$$
\underline{k}=\underline{x}+\underline{t} \circ \underline{z}^{(k)}+\left(a \underline{1}+\underline{m}^{(\underline{F})}\right) \circ \underline{n}^{(k)}=\underline{x}^{\prime}+\underline{t}^{\prime} \circ \underline{z}^{(k)}+\left(a^{\prime} \underline{1}+\underline{m}^{\left(\underline{F}^{\prime}\right)}\right) \circ \underline{n}^{(k)} .
$$

We may conclude

$$
\begin{aligned}
& (\underline{j}-\underline{i})_{K}=m_{K}^{(\underline{F})}+a=m_{K}^{\left(\underline{F}^{\prime}\right)}+a^{\prime} \\
& (\underline{k}-\underline{i})_{L}=m_{L}^{(\underline{F})}+a=m_{L}^{\left(F^{\prime}\right)}+a^{\prime}
\end{aligned}
$$

for the components with index $K=\{i, j\}$ in vector $(\underline{j}-\underline{i})$, and with index $L=\{i, k\}$ in vector $(\underline{k}-\underline{i})$, respectively. As an immediate consequence, we obtain

$$
m_{K}^{\left(\frac{F}{)}\right)}-m_{L}^{(\underline{F})}=m_{K}^{\left(F^{\prime}\right)}-m_{L}^{\left(F^{\prime}\right)} .
$$

But $M$ is a difference matrix, such that $\underline{F}$ and $\underline{F}^{\prime}$ denote the same block in $\mathcal{F}$, which contradicts our assumption.

Thus, any two blocks share at most two vertices, i.e. the constructed $(v, \mathcal{G}, \underline{\lambda})$ digraph design $\mathcal{F}^{\prime}$ is superpure as claimed.

Since $S(\mathcal{G}, \underline{\lambda})$ is eventually periodic with period $\pi$, the statement of Lemma 16 immediately implies Theorem 3 .

We append a further remark on Theorems 2 and 3: Both results ensure the existence of lower bounds $v_{D}(\mathcal{G}, \underline{\lambda})$ and $v_{S}(\mathcal{G}, \underline{\lambda})$ such that every suitable integer $v$ exceeding these bounds belongs to $D(\mathcal{G}, \underline{\lambda})$ or $S(\mathcal{G}, \underline{\lambda})$, respectively.

Usually $v_{S}$ will be much larger than $v_{D}$. A trivial lower bound for $v_{S}$ may be obtained as follows. Assume, we are given a superpure $(v, \mathcal{G}, \lambda)$ digraph design on the vertex set $V$. Let $\lambda_{\text {sum }}$ denote the sum over all components of the $r$-element 
vector $\underline{\lambda}$, and $n_{\min }(\mathcal{G})$ denote the minimum number of vertices in a member of $\mathcal{G}$. Two vertices $x$ and $y$ from $V$ occur together in at least $\lambda_{\text {sum }}$ blocks. However, the digraph design under inspection is superpure, that is, any two blocks containing $x$ and $y$ as vertices do not share any further vertex. Thus $x$ and $y$ appear together in at most $(v-2) /\left(n_{\min }(\mathcal{G})-2\right)$ blocks, which immediately implies

$$
v_{S}(\mathcal{G}, \underline{\lambda}) \geq\left(n_{\min }(\mathcal{G})-2\right) \lambda_{\text {sum }}+2 .
$$

\section{Reducible DigRAPH DESIGNS}

Throughout, let $G$ be an $r$-colored digraph with arc vector $\underline{\epsilon}(\underline{G})=\epsilon \underline{1}$ for some positive integer $\epsilon, \mathcal{G}$ be a family of $r$-colored digraphs, $\underline{\lambda}$ be an $r$-element vector of positive integers, and $\mu \geq 2$ be a positive integer. Moreover, we assume $(\mathcal{G}, \underline{\lambda})$ to be admissible.

A $(v, \mathcal{G}, \mu \underline{\lambda})$ digraph design is said to be $\underline{\lambda}$-reducible if it is the union of $\mu(v, \mathcal{G}, \underline{\lambda})$ digraph designs. 1-reducible designs, in particular, are called completely reducible. Again, we are interested in superpure designs. Let $R S(\mathcal{G}, \underline{\lambda}, \mu)$ denote the set of all integers $v$, for which a $\underline{\lambda}$-reducible superpure $(v, \mathcal{G}, \mu \underline{\lambda})$ digraph design exists. Clearly, $R S(\mathcal{G}, \underline{\lambda}, \mu)$ is a subset of $S(\mathcal{G}, \underline{\lambda})$. Similar to Lemma $8, R S(\mathcal{G}, \underline{\lambda}, \mu)$ may be proved to be PBD-closed.

Lemma 17. There exists a completely reducible superpure $(v, \underline{G}, \mu \underline{1})$ digraph design for almost every integer $v \equiv 1 \bmod \pi(\underline{G})$.

Proof. As above, let $C$ be the $r$-element set containing all colors assigned to the arcs of $\underline{G}$. Put $C^{\prime}=\left\{c^{\prime}=(c, i): c \in C, i=1, \ldots, \mu\right\}$. Next, we take $\mu$ pairwise disjoint copies of $\underline{G}$, and assign every arc of color $c$ in the $i$-th copy the new color $c^{\prime}=(c, i)$, with $c \in C$ and $1 \leq i \leq \mu$. The resultant $(\mu r)$-colored digraph is denoted by $\underline{H}$ and has arc vector $\underline{\epsilon}(\underline{H})=\epsilon \underline{1}$.

If $\epsilon$ is even, we apply the construction proposed in the proof of Lemma 7 to obtain a superpure $(q, \underline{H}, \underline{1})$ digraph design for almost every prime power $q \equiv \epsilon+1 \bmod 2 \epsilon$. When re-assigning every arc of color $c^{\prime}=(c, i)$ the original color $c$ in each of the blocks in this design, we obtain a superpure $(q, \underline{G}, \mu \underline{1})$ digraph design. Clearly, this design is the union of $\mu(q, \underline{G}, \underline{1})$ digraph designs, i.e. is completely reducible.

Afterwards, we proceed as suggested in the proof of Lemma 11. Thus, we obtain $\pi(\underline{G})$ as a period of $R S(\underline{G}, \underline{1}, \mu)$. Again, considering two disjoint copies of $\underline{G}$ gives the same result in the case of odd $\epsilon$.

In order to extend the preceding observation to arbitrary $\pi$-fibres, we need the notion of a supersimple transversal design. Let $g$ and $h \geq 2$ be positive integers. A transversal design $T D_{\mu}(h, g)$ is a family of $h$-element subsets (called blocks) of a $g h$-element set $V$, where $V$ is partitioned into disjoint $g$-element subsets (called groups) such that every block meets every group exactly once, and any two elements from different groups occur together in precisely $\mu$ blocks. The integer $\mu$ is known as the index of the transversal design. 
A transversal design of index $\mu \geq 2$ is completely reducible if it is the union of $\mu$ transversal designs of index 1. Furthermore, a transversal design is supersimple if any two different blocks share at most two elements. In [20], we proved the existence of a completely reducible supersimple transversal design $T D_{\mu}(h, q)$ for given integers $\mu$ and $h$, and all sufficiently large prime powers $q$. We shall apply this observation to prove the main result of this section.

Theorem 18. For every admissible $(\mathcal{G}, \underline{\lambda})$, every positive integer $\mu$ and almost all positive integers $v$ satisfying the necessary conditions (1) and (2), there exists a $\underline{\lambda}$-reducible superpure $(v, \mathcal{G}, \mu \underline{\lambda})$ digraph design.

Proof. To prove the claimed result, we use essentially the same ideas as in Sections 5 and 6 . Let $(\underline{G}, \underline{\lambda})$ be an assembly of $(\mathcal{G}, \underline{\lambda})$, and $\left(\underline{G}^{\prime}, \underline{1}\right)$ a refinement of $(\underline{G}, \underline{\lambda})$. As pointed out above, $\pi=\pi\left(\underline{G}^{\prime}\right)$ is a period of the eventually periodic set $R S(\mathcal{G}, \underline{\lambda}, \mu)$. Therefore, it remains to show, that for every positive integer $u$ satisfying the necessary conditions (1) and (2) the set $R S(\mathcal{G}, \underline{\lambda}, \mu) \cap \mathbb{N}_{u(\pi)}$ is non-empty, i.e. a $\pi$-fibre.

By Theorem 3, there exists an integer $w \equiv u \bmod \pi$ admitting a superpure $(w, \mathcal{G}, \underline{\lambda})$ digraph design. On the other hand, Lemma 17 and Dirichlet's theorem ensure the existence of a completely reducible superpure $\left(q, \underline{G}^{\prime}, \mu \underline{1}\right)$ digraph design for almost every prime power $q \equiv 1 \bmod \pi$. Analogously to Section 5 , we may check that this yields the existence of a $\underline{\lambda}$-reducible superpure $(q, \mathcal{G}, \mu \underline{\lambda})$ digraph design.

Now, consider a completely reducible supersimple transversal design $T D_{\mu}(w, q)$, which particularly exists for almost every prime power $q \equiv 1 \bmod \pi$. In this transversal design, we replace every block $B$ by a superpure $(w, \mathcal{G}, \underline{\lambda})$ digraph design $\mathcal{F}_{B}$, and every group $X$ by a $\underline{\lambda}$-reducible superpure $(q, \mathcal{G}, \mu \underline{\lambda})$ digraph design $\mathcal{F}_{X}$. The resultant family $\mathcal{F}$ of digraphs obviously forms a $\underline{\lambda}$-reducible $(v, \mathcal{G}, \mu \underline{\lambda})$ digraph design, where $v=w q \equiv w \equiv u \bmod \pi$ holds.

All we have to do is to verify that $\mathcal{F}$ is indeed superpure. Consider two different members of $\mathcal{F}$, and assume them to share more than two vertices. Since all families $\mathcal{F}_{B}$ and $\mathcal{F}_{X}$ are superpure, the digraphs under consideration will not belong to the same subfamily. Hence, their vertex sets are subsets of two different blocks, two different groups, or a block and a group of the transversal design. But due to the supersimplicity of the chosen transversal design, any two of these sets share at most two elements. This proves $\mathcal{F}$ to be superpure, and concludes our proof.

\section{SOME APPLICATIONS}

In this section, we collect some examples that serve to illustrate the impact of our results to known structures in design theory. To begin with, we consider supersimple designs, i.e. block designs where any two distinct blocks share at most two vertices. Supersimple $(v, 4, \lambda)$ designs have been introduced in [16], and their existence problem has been solved for $\lambda$ equal to two [16, 26], three [25] and four [1]. A supersimple $(v, k, \lambda)$ design corresponds to a superpure $\left(v, D_{k}, \lambda\right)$ digraph design, where $D_{k}$ is the (unicolored) complete digraph on $k$ vertices. In particular, we have 
$\alpha\left(D_{k}, \lambda\right)=(k-1) / \operatorname{gcd}\{k-1, \lambda\}, \beta\left(D_{k}, \lambda\right)=k(k-1) / \operatorname{gcd}\{k(k-1), \lambda\}$, and $\left(D_{k}, \lambda\right)$ is always admissible. This implies the following result.

Corollary 19. Let $k \geq 3$ and $\lambda$ be positive integers. There exists a supersimple $(v, k, \lambda)$ design for almost every $v$ satisfying the necessary conditions $\lambda(v-1) \equiv$ $0 \bmod (k-1)$ and $\lambda v(v-1) \equiv 0 \bmod k(k-1)$.

In $[16,26]$, completely reducible supersimple designs have been investigated, too. Theorem 18 gives us the following result. For $\mu=1$, i.e. in the case of completely reducible designs, the claim corresponds to an observation of Caro and Yuster [10].

Corollary 20. Let $k \geq 3, \lambda$ and $\mu$ be positive integers. There exists a $\lambda$-reducible supersimple $(v, k, \mu \lambda)$ design for almost every $v$ satisfying the necessary conditions in Corollary 19.

Next, we turn our attention to Mendelsohn designs. As pointed out in Section 1, a $(v, k, \lambda)$ Mendelsohn design is just a $\left(v, C_{k}, \lambda\right)$ digraph design, where $C_{k}$ is the directed $k$-cycle in Figure 1 . Two vertices $x, y$ are $t$-apart in $C_{k}$, if there is a directed path of length $t$ from $x$ to $y$ in $C_{k}$. A $(v, k, \lambda)$ Mendelsohn design is called perfect, if for every $t \in\{1, \ldots, k-1\}$, each pair $(x, y)$ of distinct vertices is $t$-apart in exactly $\lambda$ blocks.

For existence results and further details on perfect Mendelsohn designs, we refer to [30]. Asymptotically, the existence of perfect Mendelsohn designs was established by Zhang (see [30]). Pure perfect Mendelsohn designs have been studied e.g. in $[4,5]$ for $k=3$, and in [23] for $k=4$.
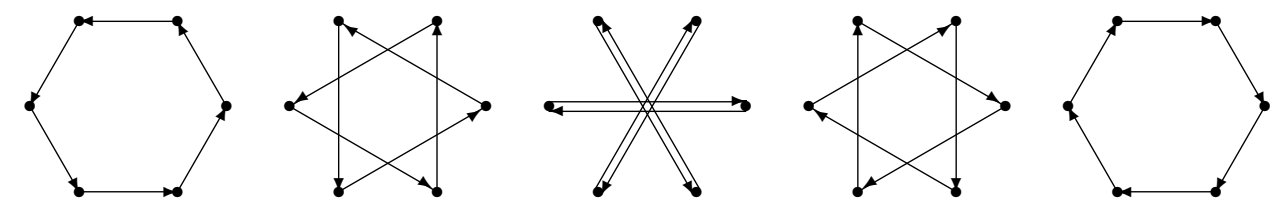

Figure 2 . The $k-1$ pages of $\underline{M}_{k}$ for $k=6$.

In order to apply our results to perfect Mendelsohn designs, we consider the $(k-1)$ colored digraph $\underline{M}_{k}$ with vertex set $V\left(\underline{M}_{k}\right)=V\left(C_{k}\right)$ where two vertices $x$ and $y$ are connected by an arc $(x, y)$ of color $t$, whenever $x$ and $y$ are $t$-apart in $C_{k}$. As an example, we included the pages of $\underline{M}_{6}$ in Figure 2. We claim that $\left(v, \underline{M}_{k}, \lambda \underline{1}\right)$ digraph designs are equivalent to perfect $(v, k, \lambda)$ Mendelsohn designs. Let $\mathcal{F}$ be a $\left(v, \underline{M}_{k}, \lambda \underline{1}\right)$ digraph design, and $\mathcal{F}_{t}$ the family of all the $t$-th pages $F_{t}$ with $\underline{F} \in \mathcal{F}$. For every $t \in\{1, \ldots, k-1\}$ and each pair $(x, y)$ we find exactly $\lambda$ digraphs $F$ containing the arc $(x, y)$ with color $t$. Hence, $x, y$ are $t$-apart in the first page $F_{1}$ of each of these digraphs. This proves the family $\mathcal{F}_{1}$ to be a perfect Mendelsohn design.

The digraph $\underline{M}_{k}$ has arc vector $\underline{\epsilon}\left(\underline{M}_{k}\right)=k \underline{1}$, and degree vector $\underline{\delta}_{u}\left(\underline{M}_{k}\right)=\underline{1}$ for all its vertices $u$. This enables us to calculate $\beta\left(\underline{M}_{k}, \lambda \underline{1}\right)=k / \operatorname{gcd}\{k, \lambda\}$ and $\alpha\left(\underline{M}_{k}, \lambda \underline{1}\right)=$ 1. Hence $\left(\underline{M}_{k}, \lambda\right)$ is always admissible, which gives rise to the following statement. A similar observation holds for simple and for pure perfect Mendelsohn designs, since the necessary conditions for their existence are essentially the same. 
Corollary 21. Let $k \geq 3$ and $\lambda$ be positive integers. There exists a superpure perfect $(v, k, \lambda)$ Mendelsohn design for almost every $v$ satisfying the necessary condition $\lambda v(v-1) \equiv 0 \bmod k$.

\section{REFERENCES}

[1] P. Adams, D. E. Bryant, and A. Khodkar. On the existence of supersimple designs with blocksize 4. Ann. of Math., 51:230-246, 1996.

[2] B. Alspach, K. Heinrich, and G. Liu. Orthogonal factorizations of graphs. In Dinitz and Stinson [14], chapter 2.

[3] F. E. Bennett and A. Mahmoodi. Directed designs. In Colbourn and Dinitz [11], chapter IV.15.

[4] F. E. Bennett and N. S. Mendelsohn. On pure cyclic triple systems and semisymmetric quasigroups. Ars Combin., 5:13-22, 1978.

[5] F. E. Bennett and H. Shen. On indecomposable pure Mendelsohn designs. Discrete Math., 97:47-57, 1991.

[6] J.-C. Bermond and D. Sotteau. Graph decompositions and G-designs. Congr. Numer., 15:53$72,1976$.

[7] T. Beth, D. Jungnickel, and H. Lenz. Design Theory. BI, Mannheim, 1985.

[8] J. Bosák. Decompositions of graphs. Kluwer, Dordrecht, 1990.

[9] P. J. Cameron and J. H. van Lint. Designs, graphs, codes and their links. University Press, Cambridge, 1991.

[10] Y. Caro and R. Yuster. Orthogonal decomposition and packing of complete graphs. J. Combin. Theory Ser. A, 88:93-111, 1999.

[11] C. J. Colbourn and J. H. Dinitz, editors. The CRC handbook of combinatorial designs. CRC press, Boca Raton, 1996.

[12] C. J. Colbourn and A. Rosa. Directed and Mendelsohn triple systems. In Dinitz and Stinson [14], chapter 4.

[13] M. Dehon. On the existence of 2-designs $S_{\lambda}(2,3, v)$ without repeated blocks. Discrete Math., 43:155-171, 1983.

[14] J. H. Dinitz and D. R. Stinson, editors. Contempory design theory. Wiley, New York, 1992.

[15] D. A. Drake. Partial $\lambda$-geometries and generalized Hadamard matrices over groups. Canad. $J$. Math., 31:617-627, 1979.

[16] H.-D. O. F. Gronau and R. C. Mullin. On supersimple 2- $(v, 4, \lambda)$-designs. J. Combin. Math. Combin. Comput., 11:113-121, 1992.

[17] H.-D. O. F. Gronau, R. C. Mullin, and P. J. Schellenberg. On orthogonal double covers of $K_{n}$ and a conjecture of Chung and West. J. Combin. Des., 3:213-231, 1995.

[18] S. Hartmann. Asymptotic results on suborthogonal $G$-decompositions of complete digraphs. Discrete Appl. Math., 95:311-320, 1999.

[19] S. Hartmann. Orthogonal decompositions of complete digraphs. Graphs Combin., 1999, to appear.

[20] S. Hartmann. On simple and supersimple transversal designs. J. Combin. Des., 8:311-320, 2000.

[21] K. Heinrich. Graph decompositions and designs. In Colbourn and Dinitz [11], chapter IV. 22

[22] P. Hell and A. Rosa. Graph decompositions, handcuffed prisoners and balanced $P$-designs. Discrete Math., 2:229-252, 1972.

[23] D. F. Hsu and H. Shen. On the existence of perfect Mendelsohn designs without repeated blocks. Ars Combin., 37:129-139, 1994.

[24] H. H. Y. Huang and N. S. Mendelsohn. Directed triple systems. J. Combin. Theory Ser. A, 14:310-318, 1973.

[25] C. Kejun. On the existence of supersimple $(v, 4,3)$ BIBDs. J. Combin. Math. Combin. Comput., 17:149-159, 1995. 
[26] A. Khodkar. Various supersimple designs with block size four. Australas. J. Combin., 9:201210, 1994.

[27] E. R. Lamken and R. M. Wilson. Decompositions of edge-colored complete graphs. J. Combin. Theory Ser. A, 89:149-200, 2000.

[28] C. C. Lindner and A. P. Street. Disjoint designs and irreducible designs without repeated blocks. Ars Combin., 21 (A):229-236, 1986.

[29] R. Mathon and A. Rosa. A census of Mendelsohn triple systems of order nine. Ars Combin., 4:309-315, 1977.

[30] E. Mendelsohn. Mendelsohn designs. In Colbourn and Dinitz [11], chapter IV.28.

[31] N. S. Mendelsohn. A natural generalization of Steiner triple systems. In Computers in number theory, pages 323-338. Academic Press, New York, 1971.

[32] D. G. Sarvate. Block designs without repeated blocks. Ars Combin., 21:71-87, 1986.

[33] U. Schumacher. Suborthogonal double covers of complete graphs by stars. Discrete Appl. Math., 95:439-444, 1999.

[34] K. Ushio. G-designs and related designs. Discrete Math., 116:299-311, 1993.

[35] R. M. Wilson. An existence theory for pairwise balanced designs II. J. Combin. Theory Ser. A, 13:246-270, 1972.

[36] R. M. Wilson. Decompositions of complete graphs into subgraphs isomorphic to a given graph. Congr. Numer., 15:647-659, 1976.

FB Mathematik, Universität Rostock, 18051 Rostock, Germany

E-mail address: sven.hartmann@mathematik.uni-rostock.de 